\title{
Ought a Four-Dimensionalist To Believe in Temporal Parts?
}

\author{
KRISTIE MILLER \\ School of Philosophical and Historical Inquiry \\ and the Centre for Time \\ The University of Sydney \\ Sydney, NSW 2006 \\ AUSTRALIA
}

\section{Introduction}

I borrow the title of this paper, slightly amended, from Parsons' recent 'Must a Four-Dimensionalist Believe in Temporal Parts?' ${ }^{1}$ Four-dimensionalism, as I use the term, is the view that persisting objects have four dimensions: they are four-dimensional 'worms' in space-time. This view is contrasted with three-dimensionalism, the view that persisting objects have three-dimensions and are wholly present at each moment at which they exist. The most common version of four-dimensionalism is perdurantism, according to which these four-dimensional objects are segmented into temporal parts - shorter lived objects that compose the four-dimensional whole in just the same way that the segments of real earth worms compose the whole worm.

According to Parsons, a four-dimensionalist need not believe in temporal parts. But ought a four-dimensionalist to believe in temporal

1 Parsons, J. 'Must a Four Dimensionalist Believe in Temporal Parts?' The Monist 83 (2000) 399-418. 
parts? This question might be thought uninteresting, insofar as it has largely been answered by force of numbers. But asking it is instructive. For there are reasons to suppose that a non-perdurantist four-dimensionalism might provide an attractive middle position between perdurantism and endurantism: ${ }^{2}$ a position that avoids many of the different counterintuitive costs associated with each of these views. So it is worth determining whether non-perdurantist four-dimensionalism is a viable theory of persistence. To that end I develop the most plausible non-perdurantist version of four-dimensionalism.

Ultimately, however, I argue that non-perdurantist four-dimensionalism does not offer a plausible middle ground position between perdurantism and endurantism. Further, in considering the reasons why non-perdurantism is unappealing, a number of problems are revealed for endurantism. Much of what is problematic about non-perdurantism is equally problematic for endurantism. What is illuminating is that the analogous problems we discover for endurantism are not those that typically constitute objections to the view. This is because evaluating non-perdurantism allows us to abstract away from two issues that cloud the debate between three- and four-dimensionalists: first, the idea that three-dimensional objects are wholly present whenever they exist, and the associated problems with cashing out this notion; and second, the idea that three-dimensional objects are strictly identical to themselves at every time at which they exist, and the attendant problems with that notion in the light of change across time. Abstracting away from these issues is fruitful, because frequently with respect to these issues, debate on either side reduces to an appeal to brute intuition leaving the debate stagnant.

That endurantism faces the same problems as non-perdurantist four-dimensionalism tells us that the debate between perdurantists and endurantists has been misplaced. We discover that perdurantism is in some sense resilient: as soon as we move away from a perdurantist to a non-perdurantist four-dimensionalism, the account exhibits fundamental problems. What is wrong with endurantism is not that it embraces identity across time, rather, what is problematic is that it is non-perdurantist. Non-perdurantist theories of persistence fail because ultimately, temporal parts are resilient: we need them in any account of persistence.

I begin by outlining some reasons to find non-perdurantist fourdimensionalism an attractive view. Then in sections three, four and five I set out a more detailed account of a non-perdurantist four-dimension-

2 I use 'endurantism' as synonymous with 'three-dimensionalism.' 
alism. This allows us, in section six, to see the shortcomings of such a position. In this section I outline a number of key objections to nonperdurantism, before in section seven arguing that analogous problems arise for endurantism.

\section{A Middle-ground Position}

This section aims to motivate the idea that a non-perdurantist fourdimensionalism - non-perdurantism as I call the view - might be attractive. To do so I will make two assumptions. First, that the key objections to endurantism and perdurantism are widely known and do not require explication here. Second, that although the details of non-perdurantism are left to section three, we have a sufficient grasp of the general idea of non-perdurantism to see, at least prima facie, why it might be thought appealing.

The general idea then, is that persisting objects are four-dimensional - they are temporally extended - but not in virtue of being composed of (maximal) temporal parts: ${ }^{3}$ non-perduring four-dimensional objects are space-time worms, but they are not segmented worms. In brief, here are some reasons why we might find the view congenial.

There are two key objections to endurantism.

(1) Endurantists can offer no substantive account of what it means for an object to be 'wholly present' at a time.

and

(2) Endurantists hold that enduring objects are strictly identical across time. But enduring objects change over time, and hence have different properties at different times. To accommodate both strict identity and change, endurantists need to appeal to

3 Thus $x$ is an instantaneous maximal temporal part of $y$ at instant $\left.t==_{\mathrm{df}} 1\right) x$ is part of $y$ and 2) $x$ exists at, but only at $t$ and 3) $x$ overlaps every part of $y$ that exists at $t$. An extended maximal temporal part of $y$ during temporal interval $T$ is an object that exists at all and only times in $T$, is part of $y$ at every time during $T$ and at every moment in $T$ overlaps everything that is part of $y$ at that moment.

4 T. Sider, Four-Dimensionalism: An Ontology of Persistence and Time (Oxford University Press 2001), 65-8. T. Merricks, 'Persistence, Parts and Presentism,' Nous 33 (1999) 421-38; M. Hinchliff, 'The Puzzle of Change,' Philosophical Perspectives 10, Metaphysics (1996) 119-33; N. Markosian, 'The 3D/4D Controversy and NonPresent Objects,' Philosophical Papers 23 (1994) 243-9. 
a dissatisfying account of the nature of intrinsic properties that involve recherché ways of temporally modifying properties or their mode of instantiation.

There are two key objections to perdurantism.

(1a) Perdurantists are committed to the implausible and ontologically profligate claim that what seems like a single persisting object is really a series of distinct short-lived and temporally overlapping $^{6}$ objects that come into and pass out of existence before our eyes.

and

(2a) Persisting objects often change properties over time. For perdurantists, this means that temporary properties are properties of temporal parts, not straightforwardly properties of the whole persisting object. So perdurantists have a dissatisfying account of the nature of intrinsic properties. ${ }^{7}$

Non-perdurantism prima facie fares better than either of these views with respect to each of these problems. The non-perdurantist can deal with (1a) by agreeing with the endurantist that an ontology of temporal parts is counterintuitive and metaphysically profligate. She can agree that where we see a single persisting object, there really does exist a single persisting object and not a myriad of temporally overlapping objects. She can concur with the endurantist in denying that persisting objects are only partly present whenever they exist, at least as long as we assume that a persisting object is partly present at a time iff some proper part of it exists at that time.

On the other hand, denying that persisting objects are only partly present whenever they exist does not entail affirming that they are wholly present whenever they exist. The non-perdurantist holds that

5 P. van Inwagen, 'Four-Dimensional Objects,' Nous 24 (1990) 245-55; S. Haslanger, 'Endurance and Temporary Intrinsics,' Analysis 49 (1989) 119-25; M. Johnston, 'Is There a Problem about Persistence?' The Aristotelian Society Supp 61 (1987) 107-35.

6 Persisting objects $A$ and $B$ temporally overlap just in case there is some time $t$, such that at $t, A$ and $B$ materially coincide. Then for the perdurantist, $A$ and $B$ temporally overlap just if either $A$ is a maximal temporal part of $B$, or $B$ is a maximal temporal part of $A$.

7 M. Hinchliff, 'The Puzzle of Change.' Philosophical Perspectives 10, Metaphysics (1996) 119-133. 
persisting objects are temporally extended, and are hence not wholly present whenever they exist, at least given a plausible interpretation of the endurantist notion. So the non-perdurantist is not committed to defending or defining the notion of being wholly present, and hence is not concerned by (1).

Moreover, the non-perdurantist denies that persisting objects are strictly identical to themselves at every time at which they exist. Like all objects, non-perduring things are strictly self-identical, but they are not strictly identical across time in the sense employed by the endurantist. Endurantists, perdurantists, and non-perdurantist four-dimensionalists all agree that persisting objects occupy ${ }^{8}$ four-dimensional regions of space. And each agrees that we can divide up a four-dimensional region of space into a series of contiguous three-dimensional regions. Call each of these three-dimensional regions a three-dimensional 'slice' of space-time. Endurantists and perdurantists both hold that if $\mathrm{R}$ is a four-dimensional region occupied by persisting object $O$, then for any three-dimensional slice of $R$, there is an object that wholly occupies that slice. ${ }^{9}$ The endurantist claim that persisting objects are strict identity over time is then the claim for any objects $O_{1}$ and $O_{2}$ that occupy slices $S_{1}$ and $S_{2}$ of $R, O_{1}$ and $O_{2}$ are strictly identical: each is $O$, wholly present, at a different time. The perdurantist claims that for any objects $O_{1}$ and $O_{2}$ that occupy slices $S_{1}$ and $S_{2}$ of $R, O_{1}$ and $O_{2}$ are distinct: each is a temporal part of $O$. The non-perdurantist also rejects the threedimensionalist's claim about strict identity over time, since for her no slice is wholly occupied by any object, so no sense can be made of the claim that what occupies one slice is strictly identical to what occupies another slice. But the sense of strict identity according to which what wholly occupies one slice is strictly identical to what occupies a distinct slice despite the fact that each of these occupants has distinct properties, is what causes problems for the endurantist and forces her to adopt recherché accounts of property instantiation. Prima facie then, one might expect the non-perdurantist to escape the need for such accounts, given that she rejects this strict identity. If that is so, then (2) is not a problem.

It is less clear exactly how the non-perdurantist fares with respect to (2a). Certainly she can agree with the endurantist that a perdura-

8 Perdurantists might think that they are identical to such regions, but set that aside for now.

9 Where an object $O$ wholly occupies a region $R$ just in case every part of $O$ occupies a region of $R$, and no proper part of $O$ occupies any region that is not a sub-region of $R$. 
ntist account of property instantiation is problematic. Since the nonperdurantist has no ontology of temporal parts, she can agree that the attribution of intrinsic properties to an object cannot be a matter of attributing that property to some part of that object. Exactly how the non-perdurantist should make sense of temporary intrinsics is more difficult. We can see, broadly speaking, how she might begin to frame such an account. Consider spatially extended mereological simples that have different properties at different space-time points or locations at which they exist. Such an extended simple might be 'half' orange and 'half' purple, despite there being no proper parts that are orange and purple respectively. Yet it is a property of the whole simple that it is orange at certain locations. By analogy, the non-perdurantist might say that the whole four-dimensional worm has properties at (spatio-temporal) locations. A sophisticated version of something like this view has been defended by Parsons, who argues for the existence of distributional properties. ${ }^{10}$ It is fair to say then, that non-perdurantism offers the possibility of resolving (2a) in a more attractive manner than perdurantism, though more work would need to be done to show exactly how property instantiation would work.

Since non-perdurantism appears to fare better with respect to (1), (2), (1a) and, possibly, (2a), this suggests it might be a viable alternative to either perdurantism or endurantism. Given this, it behoves us to develop such a view in more detail. It is to this that I turn in the following section.

\section{Terdurantism: Non-perdurantist Four-dimensionalism}

Hitherto I have talked of non-perdurantist four-dimensionalism, or just non-perdurantism. These are not perspicacious labels: they don't not tell us in what manner 'non-perduring' objects persist. Parsons describes these objects as enduring. But it is standard to think of endurance as the manner in which three-dimensional objects persist. Given this, the possibility of non-perduring four-dimensional objects suggests there are three ways of persisting. ${ }^{11}$ Call the third way terdurance. Nonperdurantist four-dimensionalists hold that persisting objects terdure,

10 J. Parsons, 'Must a Four Dimensionalist Believe in Temporal Parts?' The Monist 83 (2000) 399-418.

11 One might think there are four ways of persisting - counting the stage-view as a fourth alternative. Whether this amounts to a distinct way of persisting, i.e. by having temporal counterparts, or whether it amounts to a different semantics for four-dimensionalism is debatable. 
and hence can be thought of as terdurantists. Now I will begin to explicate terdurantism, by first considering what our world would need to be like for terdurantism to be true, and then developing the strongest version of the theory.

\section{Terdurantism, Simples and Mereological Universalism}

In what follows I make two assumptions. The first is that space-time is continuous. The second is that atomism is true: composite objects are composed entirely of simples.

Suppose there are simple objects, and composite objects are mereological fusions of simples. Then what is the nature of these simples, and under what circumstances does composition occur? Only some answers to these questions are consistent with terdurantism. I begin by arguing that the terdurantist should reject the view that simples are instantaneous, and instead hold that simples terdure.

Suppose all simples are instantaneous. Consider a set of simples all of which exist at $t_{1}$, such that there is a fusion, $S_{1}$, of those simples. Call a fusion of particulars all of which exist at all and only the same time a synchronic fusion. And suppose there exists another synchronic fusion of simples $-S_{2}-$ at $t_{2}$. Suppose our terdurantist is a mereological universalist: she holds that for every set $S$ of concrete particulars, there is a fusion of the members of $S$, where $x$ is a fusion of the members of $S$ iff every member of $S$ is part of $x$, and every part of $x$ overlaps some member of $S$. Then there exists some object $-D_{1}$ - that is the fusion of $S_{1}$ and $S_{2}$. Call an object that is the fusion of two or more synchronic fusions each of which exists at different times, a diachronic fusion. Diachronic fusions like $D_{1}$ perdure: they have synchronic fusions as maximal temporal parts. Mereological universalism guarantees that $D_{1}$ exists. So if terdurantism is the view that all persisting objects terdure, then it cannot hold both that universalism is true, and that simples are instantaneous. Indeed, the terdurantist need not endorse universalism to be forced to accept that there exist perduring objects. She need only accept that simples are instantaneous, and that cross-temporal composition is mereological: persisting objects are fusions of synchronic fusions or of instantaneous simples across time. This entails that persisting objects perdure.

The terdurantist could endorse the view that simples are instantaneous, but hold that composition across time is not mereological. ${ }^{12}$ That is, persisting objects are not related to synchronic fusions as wholes to

12 I have elsewhere defended an account of non-mereological universalism that 
parts, but rather, are related to them in some other manner. An endurantist could appeal to the constitution relation, holding that enduring objects - which are strictly identical across time - are nevertheless constituted at different times by different synchronic fusions. Presumably the terdurantist could employ a similar relation to analogous effect. Nevertheless, even having made this move the terdurantist would still need to endorse the claim that whenever there exists a terduring object $O$, at every time $t$ at which $O$ exists, there exists some instantaneous object $I$ (a synchronic fusion) that materially coincides with $O$ at $t$. I would not be a proper (temporal) part of $O$, so this is consistent with $O$ terduring. However, on this view terdurantism would not be preferable to perdurantism with respect to (1a). The terdurantist ontology would look just like the perdurantist's, it would merely be that what appear to be segments of a terduring object are not in fact parts of that object.

So a plausible terdurantism requires some background assumptions about the furniture of the universe: namely that simples are not instantaneous. In order to make the strongest case for terdurantism, in what follows I make that assumption.

\section{Terduring Simples and Composites}

Intuitively, terduring simples are four-dimensional partless objects that are not strictly identical across time. Since they are both four-dimensional and simple, they clearly do not perdure, and since they are not strictly identical across time, they do not endure. Then:

A simple $S$ terdures iff: (i) $S$ is mereologically simple and (ii) $S$ persists through some temporal interval $T$ which contains instants $t$ and $t^{*}$ and (iii) the three-dimensional slice of $S$ at $t$ is not strictly identical to the three-dimensional slice of $S$ at $t^{*}$.

Then it seems natural to think of composite terduring objects as fusions of terduring simples. There are, however, two matters to address before we can define terdurance for composites. The first concerns exactly what the 'non-perdurantist' aspect of terdurantism amounts to. The second concerns how we should understand primitive parthood if composite terduring objects are fusions of terduring simples. I consider these matters in order.

Suppose a terduring simple $S_{1}$ persists through interval $T_{1}$, and terduring simple $S_{2}$ persists through interval $T_{2}$, such that $T_{1}$ and $T_{2}$ do not

explicates this latter claim. See K. Miller, 'Non-Mereological Universalism,' European Journal of Philosophy 14 (2006) 427-45. 
overlap. ${ }^{13}$ If mereological universalism is true, then there is a fusion of $S_{1}$ and $S_{2}$. In some good sense, we might say, that object perdures. It has two proper maximal temporal parts: $S_{1}$ and $S_{2}$. I take it that this is not something that should worry the terdurantist. It is a little unexpected if it turns out that strictly speaking such an object counts as both perduring and terduring. But the existence of this object is not antithetical to the non-perdurantist motivations of terdurantism. After all, anyone who accepts that there can be fusions of objects that exist at wholly distinct times, will have to concede that in this sense of perdurance, these objects perdure. The terdurantist is at pains to deny a rather richer sense of perdurance: the sense in which at every time at which a persisting object exists, it exists at that time in virtue of the existence of some instantaneous maximal temporal part at that time..$^{14}$ It is also at pains to deny that for any sub-interval during which a persisting object exists, there exists some extended maximal temporal part of that object during and only during that sub-interval. In essence, she denies what I'll call the rich perdurantist thesis (RPT):

RPT: For any persisting object $O$ that exists during interval $T$, and for every instant $t$ in $T$, and every sub-interval $I$ in $T$ (i) there is some object $O_{1}$ that exists at and only at $t$, (ii) there is some object $\mathrm{O}_{2}$ that exists during and only during $I$, (iii) $\mathrm{O}_{1}$ overlaps every part of $O$ at $t$, (iv) $O_{2}$ overlaps every part of $O$ during $I$ and (v) $O_{1}$ and $\mathrm{O}_{2}$ are proper parts of $\mathrm{O}$.

Since the fusion of terduring simples $S_{1}$ and $S_{2}$ does not entail the truth of RPT, the terdurantist need not be worried by the prospect of such an object.

This brings us to the second matter for concern: primitive parthood. It is natural to take the primitive parthood relation between terduring wholes and their parts to be temporally unmodified (as the perdurantist does) so that the primitive notion is ' $P$ is part of $O$, ' not ' $P$ is part of $O$ at $t .^{15}$

To see why, consider the thesis of mereological universalism. Considered in a temporally unmodified way, mereological universalism is the thesis that for any arbitrary set of particulars, there is a fusion of the

Or, in a world where space-time is discrete, that a persisting object exists during each smallest interval, in virtue of some maximal part existing at that interval.

15 Then $y$ is a fusion of the $x \mathrm{~s}$ iff every one of the $x \mathrm{~s}$ is a part of $y$, and no part of $y$ fails to overlap at least one of the $x \mathrm{~s}$. 
members of that set. If temporally modified parthood is primitive we fuse particulars at times. There are two different ways of thinking about primitive temporally modified parthood. Roughly, the first of these applies the temporal modifier to the particulars. The second applies the temporal modifier to the fusion relation. So, if $O_{1}$ and $O_{2}$ are persisting objects that exist at $t$, then the first has the form ' $\mathrm{O}_{1}$-at- $t$ and $O_{2}$-at- $t$ are fused.' The second has the form ' $O_{1}$ and $O_{2}$ are fused-at-t.' Shortly I will argue in detail that the former should be unattractive to the non-perdurantist. But in a sense we can see why this view will be problematic just be looking at the form of the claim. For it seems to require that there is some entity, $\mathrm{O}_{1}$-at- $t$ that can be fused, but if that entity exists, it looks for all the world as though it is a temporal part of $O_{1}$. So the idea in the argument that follows is that understanding temporally modified parthood in this way is to do something akin to presupposing that persisting entities have temporal parts.

If we understand temporally modified parthood as temporally modifying particulars, then universalism is the thesis that for any arbitrary set of particulars-at-times, there is a fusion of the members of that set.

Let us say that $y$ is a fusion of the $x \mathrm{~s}$ at $t$ iff every one of the $x \mathrm{~s}$ is a part of $y$ at $t$ and no part of $y$ fails to overlap at least one of the $x \mathrm{~s}$ at $t$. Given universalism, for any arbitrary xs at $t$ there is some fusion, $y$, of the $x \mathrm{~s}$ at $t$ : there is some instantaneous object $y$, that exists at and only at $t$, and which has each of the $x \mathrm{~s}$ at $t$ as parts. If universalism guarantees that $y$ exists, then it guarantees that for every persisting object $O$ and any arbitrary time $t$ at which $O$ exists, there is some instantaneous object $O^{*}$ that overlaps every part of $O$ at $t$ : for it guarantees that there is a fusion $\left(O^{*}\right)$ of the $x \mathrm{~s}$ at $t$ that are parts of $O .{ }^{16}$ But this is very close to endorsing perdurantism. It means that if we have a persisting object $O$, we also have an object $O$-at- $t$ that completely overlaps $O$ at $t$, and so forth for every time that $O$ exists. Conceding this would not force the terdurantist to embrace RPT, though it would entail that she embrace clauses (i) and (iii). What allows her to avoid the rich perdurantist thesis is that she can maintain that the instantaneous objects that overlap persisting objects at times are not proper parts of those persisting objects - clause (v) does not hold. This, however, is fairly unsatisfactory. Although in spirit the terdurantist rejects RPT, she accepts those aspects of it that would preclude her from having a suitable response to objection (1a). It

16 In fact, that outcome seems largely independent of whether we endorse universalism or a more restricted composition. Plausibly, whatever restrictions we place on composition will be consistent with there existing fusions of the $x s$ at $t$ where the $x s$ at $t$ all overlap a single persisting object at $t$. 
would therefore be unclear what advantage this form of terdurantism would have over perdurantism proper.

Worse, if she does accept universalism this entails that for each subinterval at which a persisting object exists, there is a fusion of each of the synchronic fusions that overlap that object during that interval. So while the restrictivist might get away with endorsing only clauses (i) and (iii) of RPT, the universalist must also endorse (ii) and (iv). Furthermore, universalism entails that for any persisting object, there is a fusion of all of the instantaneous objects that overlap that object at some time. So it entails that for every terduring object, that object wholly overlaps some perduring object: that is, for any terduring object $O$ with temporal extent $T$, there is some perduring object $P$ with temporal extent $T$, such that at every instant $t$ in $T, P$ overlaps every part of $O$ at $t$. This seems most unappealing. The instantaneous objects that are the temporal parts of $P$ need not be temporal parts of $O$, thus allowing the terdurantist to reject clause (v) and hold that $O$ is not identical to $P$. But that hardly makes the view any more attractive, since it essentially endorses perdurantism, and adds an additional ontological layer: terduring objects. Given this, the terdurantist should reject the idea that primitive parthood is temporally modified in this manner.

Indeed, it is easy to see why the terdurantist should think that primitive parthood is not modified in this way. By analogy, we could hold that there is a primitive spatially modified parthood relation such that we can fuse particulars at spatial locations. Then universalism is the thesis that for any arbitrary set of particulars at locations, there is a fusion of the members of that set. In a world in which there exist spatially extended simples, this would entail that there is something that has as parts, 'chunks' of those simples - those simples at locations. But if a simple $S$ occupies region $R$, then there is nothing in sub-region $R^{*}$ that could be a part of some further object that is a fusion of, among other things, $S$ at $R^{*}$. Mutatis mutandis for simples at times.

The other option for making sense of temporally modified parthood is to apply the temporal modifier to the fusion relation. Thus we have the claim that particulars are fused-at-times. Prima facie, this view looks like it might be more amenable. Instead of talking about fusions simpliciter, we talk about F-at- $\mathrm{t}_{1}, \mathrm{~F}-\mathrm{at}-\mathrm{t}_{2}$, and so forth. This means we are not committed to any of the parts of some fusion-at- $t$ themselves being instantaneous objects, nor to F-at-t itself being an instantaneous object. Hence the threat of tacit committed to the existence of temporal parts evaporates. It also explains how objects can gain and lose parts over time, since the parts of F-at- $t_{1}$ might be different to the parts of F-at- $\mathrm{t}_{2}$.

So, we might say that F-at-t fuses the $x_{s}$ iff every $x$ is part of F-at-t, and no part of F-at-t fails to overlap at least one of the $x s$ at $t$. But this 
is problematic. Suppose some non-perduring object $O$, is one of these xs. Is it true that $O$ is part of F-at-t? Surely all of F-at-t's parts exist at, and only at $t$. Otherwise there is no sense in which the fusion relation is temporally modified. But it is not true that $O$ simpliciter is part of F-at-t. It looks much more like $O$ properly overlaps F-at-t. $O$ 's parts at $t$ might be parts of F-at-t, but $O$ has other parts at other times that are not parts of F-at-t. Notice that even if we amend the definition by adding in a further temporal index it will not help. Suppose we say that F-at- $t$ fuses the $x s$ iff at $t$ every $x$ is part of F-at- $t$ and no part of F-at- $t$ fails to overlap at least one of the $x s$ at $t$. It is still not true, at $t$, that $O$ is part of F-at- $t$. Rather, what we want to say is, roughly, that 'the bit' of $O$ that exists at $t$ is part of F-at- $t$. Even if we modify the fusion relation, we still need to apply a temporal index to $O$ itself. The worry is that once we apply that temporal index, talk of fusing $O$, at $t$, is tantamount to talk of fusing $O$ at-t. Suppose we try to cash out the idea of fusing $\mathrm{O}$, at $\mathrm{t}$ by reference to $\mathrm{O}$ 's proper parts at $\mathrm{t}$, rather than to talk of $\mathrm{O}$-at-t. Then we say that $\mathrm{F}$-at- $t$ fuses $O$, at $t$, iff every proper part of $O$ that is located at $t$, is part of F-at- $t$. The problem is that this just moves the bump in the carpet. Consider the proper parts of $O$ that are located at $t$ and are parts of F-at- $t$. If these parts of $O$ are non-perduring objects, then they are not parts of F-at-t. For the same reason that $O$ is not part of F-at- $t$. The only way we can make sense of fusing $O$, at $t$, without it seeming as though we are really fusing $O$-at$t$, is to say that F-at- $t$ fuses $O$, at $t$ iff there is a complete decomposition of $O$ at $t$, such that every part in that decomposition is part of F-at-t. The idea is that the parts in the relevant decomposition are parts, simpliciter of F-at-t and this is how we make sense of the idea that $O$, at $t$, is a part of F-at- $t$. But notice that this in turn requires that there exist instantaneous objects, namely the parts that are in the relevant decomposition of $O$ at $t$. For only instantaneous objects could be parts, simpliciter, of F-at-t. But if there are such instantaneous objects, then mereological universalism will guarantee that there is a fusion of them, and will thus return us to endorsing something like perdurantism.

Thus the non-perdurantist should reject the idea that primitive parthood is temporally modified. That does not mean that we cannot define a notion of temporally modified parthood for terduring objects. Imagine a world $w_{1}$ in which there exist only three terduring simples, $x, y$ and $z$. Suppose that $x$ terdures from $t_{1}$ to $t_{5^{\prime}} y$ terdures from $\dddot{\omega}_{\omega_{\omega}}$ to $t_{6^{\prime}}$ and $z$ terdures from $t_{3}$ to $t_{7}$. Suppose mereological universalism is true. Then in $w_{1}$ there exist four fusions: $x y, x z, z y$, and $x y z$. Let us stipulate that these fusions are composite terduring objects. ${ }^{17}$ Then there are times at which 
$x y z$ exists $\left(t_{7}\right)$, and $x y$ does not. ${ }^{18}$ So there is a sense in which terduring objects have particulars as parts at some times and not others. We can define temporally modified parthood from the primitive notion, such that $P$ is part of $F$ at a time $t$ just if $P$ is part of $F$, and ' and $F$ exist at $t$. Then talk of the parts of a fusion at a time is just talk of its parts simpliciter, that exist at that time, not talk of something that is a part-at-a-time of that is a fusion-at-a-time, of a part.

Having sorted out these two matters, we can now move on to think about composite terduring objects. Here is one possibility:

A complex object $O$, terdures iff: (i) $O$ persist through some temporal interval $T$ which contains temporal instants $t$ and $t^{*}$ and (ii) the three-dimensional slice of $O$ at $\mathrm{t}$ is not strictly identical to the three-dimensional slice of $O$ at $t^{*}$ and (iii) it is not the case that for every instant $t$ in $T$ and sub-interval $I$ in $T$, that there is some object $O^{*}$ that exists at exactly that instant or sub-interval, and which overlaps every part of $O$ at that instant or during that sub-interval.

Clauses (i) and (ii) simply tell us that an object terdures if it persists and is not strictly identical across time. Clause (iii) tells us that terduring objects are not temporally segmented into maximal temporal parts: it tells us that the rich perdurantist thesis is false of terduring objects. Notice that this is a fairly strong version of terdurantism, for it entails that the rich perdurantist thesis is false not just because clause (v) of that thesis fails to hold - the relevant overlapping objects fail to be parts of the terduring object. Rather, it is false because there are no such overlapping objects: clauses (i) - (iv) are all false. A weaker kind of terdurantism might amend clause (iii) of the definition to read: it is not the case that for every instant $t$ in $T$ and sub-interval $I$ in $T$, that there is some object $O^{*}$ that exists at exactly that instant or sub-interval, and which overlaps every part of $O$ at that instant or during that sub-interval, such that $O^{*}$ is a proper part of $O$. This would allow that clauses (i) - (iv) of the RPT could be true of some terduring object, but that cause (v) must be false. Since I take it that this is a less attractive view, I will stick with the stronger version.

consistent with them being three-dimensional objects: for these fusions could be strictly identical across time despite the fact that their parts are not.

18 I assume that a persisting fusion exists at a time just as long as it least one its parts exists at that time. 
Then it seems plausible that terduring composite objects are fusions of terduring simples. Let us say that $F$ is a terduring fusion iff $F$ is a fusion of terduring simples. If primitive parthood is temporally unmodified, it is relatively easy to see why any terduring fusion will meet all of the clauses above. Matters are not, however, so straightforward. As we will see in the next section, ordinary objects do not seem to be terduring fusions.

\section{Fusions and Non-fusions}

In the actual world objects lose parts. An object might lose a part because the particular that is that part ceases to exist. Call this the weak sense of losing a part. A persisting object $O$ loses a part $P$ in this weak sense iff (i) $P$ is part of $O$ and (ii) there is some time $t$ at which $O$ exists, and $P$ does not. Alternatively, an object might lose a part because the particular that is that part simply ceases to be related part-wise to the object. Call this the strong sense of losing a part. A persisting object $O$ loses a part $P$ in a strong sense iff (i) there is some time $t$ at which $P$ is part of $O$, and (ii) there is some time $t^{*}$ at which both $P$ and $O$ exist, and (iii) at $t^{*} P$ is not part of $O$.

Composite objects lose parts in both of these ways. But no fusion of terduring simples loses parts in the strong sense. By definition, if $P$ is part of $O$, then for any time at which $P$ and $O$ exist, $P$ is part of $O$ at that time. That is what it is for parthood to be temporally unmodified. This suggests that everyday objects are not fusions of terduring simples. That claim makes good sense if we return to consider world $w_{1}$ in which there exist $x, y$ and $z$ and the different fusions thereof. Though there are only four possible fusions in $w_{1}$, it seems conceivable that there could be an object, $O_{1}$, that exists between $t_{2}$ and $t_{5}$ and has spatial parts $x$ and $y$ at $t_{2^{\prime}} y$ and $z$ at $t_{3^{\prime}} x$ and $z$ at $t_{4^{\prime}}$ and $x, y$ and $z$ at $t_{5}$. But $O_{1}$ is not the fusion of $x, y$ and $z$ : that object exists from $t_{1}$ to $t_{7}$. Unlike a fusion, $O_{1}$ strongly loses parts: it has $x$ as a part at $t_{2}$, but at $t_{3} x$ exists and is not part of $O_{1}$. Yet if $O_{1}$ terdures and primitive parthood is not temporally modified, then it cannot be the fusion of $x$ and $y$ at $t_{2}, y$ and $z$ at $t_{3}$ and so forth. If that is true, then there is good reason to suppose that many actual persisting objects are, like $O_{1}$, non-fusions: persisting objects that are not fusions of terduring simples.

Given our definition of terduring composites, however, that $O_{1}$ is not a fusion of terduring simples does not entail that it is not a terduring object, only that it is not a terduring fusion. So what is $O_{1}$, and what is the relation between $O_{1}$ and the fusions in $w_{1}$ ? Let us say that $O_{1}$ is a terduring non-fusion, where $O$ is a terduring non-fusion iff (i) $O$ is a composite terduring object and (ii) $O$ is not a fusion of terduring simples. 
What is the relation between $O_{1}$ and the fusions in $w_{1}$ ? At $t_{2^{\prime}} O_{1}$ and $x y$ share the same parts. How do non-fusions have parts? They appear to have parts at times, but the notion of parthood at a time is defined in terms of temporally unmodified parthood, and there is no sense in which non-fusions have parts in this latter sense. Plausibly, the answer resides in whatever relation it is that holds between $O_{1}$ and $x y$ at $t_{2}$. Presumably the idea will be that non-fusions in some sense 'inherit' their 'parts' from the fusions to which they are related at times: a non-fusion $O$ has a part $P$ at $t$ just if at $t, O$ is related to some fusion $F$ that has $P$ as a part at $t$. Clearly we cannot define this relation, on pain of circularity, in terms of parthood.

Call the relation that holds between terduring fusions and non-fusions at times, the compilation relation. Then we might say that compilation occurs just when a fusion and non-fusion spatially coincide. ${ }^{19}$ Unfortunately, the possibility of inter-penetrable matter (distinct particles that can temporarily exist at all of the same space-time points) makes that implausible, since we do not want to say that such particles are related by compilation. The difference between a case of inter-penetration and one of compilation seems to be the intuition that had the fusion not existed at the relevant time, then the non-fusion would not have existed at that time either, while the same is not true of inter-penetrable particles. In some sense the non-fusion only exists because the fusion does. So we might say that what it is for a non-fusion to exist at a time, is to be compiled by a fusion at that time. Not so for inter-penetrable particles. Suppose particles $P$ and $P^{*}$ are inter-penetrable and that they spatially coincide at time $t$ and location $L$. Then there is a robust sense in which had $P$ not existed at $t$ and $L, P^{*}$ would still have existed at that time and location. ${ }^{20}$ The existence of $P$ is independent of the existence of $P^{*}$, it is contingent that they spatially coincide.

19 Where to spatially coincide is not simply to exist within the same spatial boundary, but to exist at all and only the same spatial points. Notice that we cannot define compilation in terms of material coincidence at a time on pain of circularity, since that is in turn defined in terms of parthood at a time.

20 Actually this is not quite right. Even if we set aside worries about deviant causal chains, if it turns out that $P$ and $P^{*}$ have some sort of charge, then it might be the case that had $P$ not existed at the relevant time and location, then although $P^{*}$ would still have existed, it would have existed at a slightly different location. In fact, this might even be nomologically necessary. Then there is no robust sense in which had $P$ not existed at $L$ at $t$, then $P^{*}$ would still have existed at $L$ at $t$. Then to capture the sense in which $P$ and $P^{*}$ are independent, we could say something like the following: $P$ and $P^{*}$ are independent and distinct just if there is some world that is identical to our world up to time $t$, such that when at $t$, God makes it the case that $P$ does not exist, it is still the case that $P^{*}$ exists at $t$ at $L$. The idea here is 
This suggests that a fusion compiles a non-fusion at a time if the existence of the fusion at that time entails the existence of the non-fusion at that time. Hence:

Compilation: A fusion $F$ compiles a non-fusion $O$ at a time $t$ iff (i) $F$ and $O$ exist at $t$, and (ii) The existence of $O$ at $t$ is entailed by $F$ existing at $t$ and $F$ having the intrinsic properties at $t$ that it does and (iii) there is no proper part of $F$ whose existence entails the existence of $O$ at $t^{21}$

This definition captures the sense in which the truth maker for the claim that there exists a non-fusion at $t$ is that there exists some fusion at $t$, with a particular set of intrinsic properties at $t$, such that the existence of that fusion with those properties entails the existence of the non-fusion at that time. In what follows I will say nothing about under what circumstances fusions at times compile non-fusions. Presumably they do not always do so (though it is consistent with terdurantism that they do). As I just defined compilation, the existence of the relevant fusion, with the intrinsic properties it has, is sufficient for the existence of a non-fusion at a time. This is a nice simplifying assumption that I will accept for the remainder of the paper. Of course, one might think that other factors are relevant to whether some non-fusion exists at a time, such as which other non-fusions exist at that time, and what relations holds between these non-fusions. Then the definition above could be amended to include whatever other features one has in mind. ${ }^{22}$

just that at $t$, God could make it the case that $P$ does not exist, yet $P^{*}$ would still exist at the same location. I thank David Braddon-Mitchell for this suggestion.

21 Notice that clause (iii) is required in order to prevent its being the case that, for instance, fusion $x y$ compiles at $t_{2}$ some non-fusion $\mathrm{O}_{2}$ that has spatial part $x$ as an improper part at $t_{2}$.

22 For a defense of something like this position see K. Miller, 'Thing and Object' Acta Analytica (forthcoming). One might worry that if enough 'contextual' or 'conventional' sorts of features are included in the definition of a non-fusion, this might lead us to eliminativism about ordinary objects. The idea, perhaps, is that ordinary objects only really exist if their properties are independent of our conventions. It seems to me that this is a controversial claim about what is required to vindicate realism, and certainly not one that a realist must endorse. Something might count as a work of art only if certain artistic conventions hold. So the existence of a work of art might in part supervene on the existence of these conventions. But, as I see it, conventions are perfectly real thing themselves - they supervene on the behaviours of persons and institutions over time - they are not, 
This notion of compilation requires that one embrace a sort of modified Humeanism about necessary connections between distinct existences. Unlike straight Humeanism, it allows that such connections do exist - between compiling and compiled objects. On the other hand such connections only exist between compiling and compiled objects. We do not want it to be the case that there could exist a necessary connection between a fusion at location $L$, and a non-fusion at location $L^{*}$, since we do not want this to count as a case of compilation. We could introduce an extra clause into the definition, so that a fusion $F$ compiles a non-fusion $O$ at $t$ only if at $t, F$ and $O$ spatially coincide. But suppose $F_{1}$ is a fusion of inter-penetrable particles and non-fusion $O_{1}$ has inter-penetrable particles as parts at times. Suppose further that at $t_{1} F_{1}$ and $O_{1}$ spatially coincide. Then it is consistent with the amended definition that $F_{1}$ compiles $O_{1}$ at $t_{1}$. The point is that it is not spatial coincidence or lack thereof that marks out the difference between compilation and a failure of compilation: what marks out the difference is whether the entities in question are genuinely independent or not. If $F_{1}$ does not compile $O_{1}$ at $t_{1}$, it is because its existence at that time does not entail the existence of $\mathrm{O}_{1}$ at that time. This tells us that cases of compilation exhaust all and only the necessary connections between distinct objects.

Where does that leave us? In essence, the terdurantist holds that there exist various fusions of terduring simples: terduring fusions, each of which are related to their parts by a temporally unmodified parthood relation. Ordinary objects are not related to their parts in this way; nor are they fusions of parts at times. Rather they are related, by compilation, to different terduring fusions at different times, and they have as parts at those times, the parts of the fusion that compile them at that time.

So we have an account of how terduring non-fusions are related to terduring fusions at times. That still leaves the question of what to say about cases where two or more non-fusions (such as persons and bodies) coincide at times. Plausibly, the terdurantist will say that what it is for two or more non-fusions to materially coincide at a time, is to be related in the same way to a fusion that compiles each of them at that time. Call this relation the co-compilation relation. Hence:

in most cases, mere decisions of fiat. So it is not clear why having conventions in this sense in the supervenience base for the existence of objects ought to lead us to be eliminativists about those objects. Of course, I cannot argue for this here, and since for the remainder of the paper I do not include such features in the definition of a non-fusion, we can set the issue aside. After all, this worry is really orthogonal to whether terdurantism is true. 
Co-compilation: For any two or more non-fusions $O$ and $O^{*}, O$ and $O^{*}$ are co-compiled at $t$ just if the fusion that compiles $O$ at $t$ also compiles $O^{*}$ at $t$.

Then materially coinciding non-fusions are not directly related, rather, they are related indirectly in virtue of both being related to some third object, the fusion that compiles each of them at some time.

As I see it, the view just outlined is the strongest version of terdurantism. In the next section I will argue that it faces a number of problems.

\section{Problems for Terdurantism}

\section{Kinds of Objects}

Terdurantism is committed to the existence of two kinds of persisting objects: fusions and non-fusions. If not problematic, one might suppose that is at least unattractive.

That is perhaps a little hasty. Strictly speaking nothing about terdurantism entails that any non-fusions exist. It is a further claim of the terdurantism I have described that it holds that certain fusions compile non-fusions. But the motivation for this claim is strong: everyday objects are not fusions of terduring simples, for they strongly gain and lose parts. ${ }^{23}$ Now, one might simply bite the bullet here, and be elimina-

23 One might be tempted to say something else. Namely, that the appearance of strong part loss is just that, mere appearance. Perdurantists might seem to be making that claim. After all, perduring objects are mereological fusions of temporal parts, and hence, do not strongly lose any parts. But some perduring objects are ordinary objects. So if the perdurantist can have ordinary objects without strong part loss, maybe the terdurantist can too. In that case, the terdurantist might not need nonfusions in her ontology.

But this will not work. Why does it seem as though ordinary objects strongly lose parts if perdurantism is true? Well, consider a perduring object $O$ that exists at $t_{1}$ and $t_{2}$. It is the fusion of temporal parts $\mathrm{O}$-at- $\mathrm{t}_{1}$ and $\mathrm{O}$-at- $\mathrm{t}_{2}$. It never gains or loses these parts: it has them both tenselessly. O-at- $t_{1}$ has spatial parts: $P_{1}$ and $P_{2}$. O-at- $t_{2}$ has spatial parts $\mathrm{P}_{2}$ and $\mathrm{P}_{3}$. O-at- $t_{1}$ never loses any spatial part, and neither does $\mathrm{O}$ at $\mathrm{t}_{2}$ (obviously, since they have no duration in which to lose anything). But there is some sense in which $\mathrm{O}$ does lose a part, namely $\mathrm{P}_{1}$. And this is not a mere 'seeming.' Suppose $\mathrm{P}_{1}$ persists. The temporal part of $\mathrm{P}_{1}$ that is part of $\mathrm{O}$-at- $\mathrm{t}_{1}$ does, not exist at $t_{2}$. This is why, strictly speaking, $O$ does not lose a part: because there is no wholly present object that exists at $t_{1}$ and is part of $O$ at $t_{1}$, and which exists at $t_{2}$ and is not part of $\mathrm{O}$ at $\mathrm{t}_{2}$.

But when we say that $\mathrm{O}$ loses a part we can plausibly be held to be making the following claim. If perduring object $\mathrm{O}$ has perduring object $\mathrm{P}_{1}$ as a (spatial) part 
tivist about non-fusions. Strictly speaking composition is mereological, and the only composite objects that exist are fusions. But we sometimes talk as though there exist some other objects: objects that are arrangements, at different times, of different fusions. But we could translate any such talk into talk about fusions at times. I cannot say that I find this line promising. Eliminativism about everyday object does not strike me as a particularly plausible view. So while embracing eliminativism would dissolve the need for two distinct kinds of entity, it could equally be counted as a significant cost to terdurantism.

Assuming that one is not an eliminativist about everyday objects, the terdurantist is committed to the existence of both fusions and nonfusions. This is no fatal objection. But it is odd. It is especially odd when one considers the natures of the objects in question. For we might have tended to think of ordinary objects as the paradigm objects, and odd gerrymandered objects as merely fusions of those ordinary objects at times and places. For the terdurantist though, the basic persisting composite objects are fusions. Depending on the longevity of the terduring simples of which these composites are composed, the composites might be long-lived things that are most of the time massively spatially scattered and not at all object-like. It is entirely conceivable that actually, there are various fusions of simples many of which existed at the time of the big bang, most of which were scattered throughout space for millions of years, and which briefly coalesced on earth for a short period before dispersing again into the cosmos. It is when these simples

at some time (and hence $\mathrm{O}$-at-t has $\mathrm{P}_{1}$-at-t as a part simpliciter), then $\mathrm{O}$ counts as strongly losing $P_{1}$ if $P_{1}$ exists at a time other than $t_{1}$ in virtue of a $t_{n}$ part existing at $t_{n}$, such that the $t_{n}$ part of $P_{1}$ is not a part of any temporal part of $O$. The point is just that ordinary talk about part loss has to be reconstrued as talk about perduring parts. Once understood that way, then perduring objects are such that at one time some perduring object is part of them, and at another time that perduring object is not part of them. This is what makes ordinary talk about part loss true.

Now, more to the point, the terdurantist cannot tell any such story. To put it another way, because the perdurantist has access to instantaneous or short-lived temporal parts, for any ordinary object I describe, such that I claim that that object has different parts at different times, the perdurantist can 'construct' a perduring object that has just those parts at just those times, by being composed of the relevant temporal parts at those times. If $\mathrm{I}$ say that $\mathrm{O}$ has $\mathrm{P}$ at $\mathrm{t}_{1}$ and loses $\mathrm{P}$ at $\mathrm{t}_{2}$ then the perdurantist will say that $\mathrm{O}$ is a fusion of parts that includes $\mathrm{P}$-at- $\mathrm{t}_{1}$, and does not include P-at- $t_{2}$. But the terdurantist does not have the apparatus to do this. If $\mathrm{P}$ is a non-perduring object, then any fusion that includes $\mathrm{P}$ will always include $\mathrm{P}$, so there is no sense at all in which a fusion can lose $\mathrm{P}$. Whereas if $\mathrm{P}$ is a perduring object, then a fusion can include $P$-at- $\mathrm{t}_{1}$, but not $\mathrm{P}-\mathrm{at}-\mathrm{t}_{2}$ and hence the perdurantist can make sense of an object like $\mathrm{O}$, whereas the terdurantist cannot. At least, she cannot if $\mathrm{O}$ is supposed to be a fusion. 
thus coalesce, that we are inclined to say that their fusion compiles, at a time, some non-fusion. So the picture is one in which our basic ontology might involve objects that are for most of their existence spatially scattered. Everyday objects are not part of this basic ontology, rather, they come into existence just on the occasions when the basic fusions compile some non-fusion at a time. ${ }^{24}$

While that is perfectly coherent, it is not the usual picture we have in mind when we think of composition and objecthood, particularly given that for the terdurantist, it is fusions that are ontologically basic. Non-fusions exist at times solely in virtue of being related to fusions by the compilation relation. Notice that this means that we need a lot of fusions. The terdurantist might not be a universalist, but regardless of how she restricts composition, if she is not to be an eliminativist about ordinary objects she will need to admit into her ontology enough fusions to compile the non-fusions whose existence we are committed to. And since normal composite objects gain and lose a lot of parts across time, this means they are compiled by different fusions at many different times at which they exist. Ultimately, then, the terdurantist is committed not only to the existence of two quite different kinds of

24 There are two rather distinct worries here. One is that fusions turn out to be more ontologically basic than objects rather than the other way around, and the other is that the fusions there are, are very unlike any of our ordinary objects. I think it is the combination of these two claims that is unattractive. While it is no part of perdurantism that fusions are ontologically more basic than objects, some perdurantists do hold this additional thesis. This perdurantist thesis about the direction of fundamentality is that parts are more fundamental than wholes, and hence that temporal parts are more fundamental that persisting objects. This is a rather different claim to the claim the terdurantist makes about the direction of fundamentality, which is that fusions, one kind of object, are more basic than nonfusions, a different kind of object that supervenes on the former. But one might think that the terdurantist is no worse off in making her claim about the direction of fundamentality than is the perdurantist in making her somewhat different claim. I think the force of the objection, though, lies first in the fact that the perdurantist claim about fundamentality is purely optional, whereas the terdurantist claim about fundamentality is not, and second, that even for perdurantists who accept the fundamentality claim, at least some of the parts they take to be fundamental are pretty ordinary. If mereological universalism is true, then some fusions are odd, and there's no getting around that. But temporal parts of ordinary objects are pretty ordinary - they are things that look just like ordinary objects at a time. This is not the case for the terdurantist: she has nothing in her fundamental ontology of fusions that looks like an ordinary object. All fusions turn out to be odd, gerrymandered or scattered entities, and only non-fusions are like ordinary objects. Perhaps it is controversial the extent to which this is a cost of terdurantism, but certainly there are very real differences between terdurantism and perdurantism to be found here. 
objects, but also to the existence of a lot of odd scattered objects: the many terduring fusions that compile everyday objects at times.

It is difficult to evaluate exactly to what extent terdurantism falls foul of the objection to perdurantism we find in (1a). (1a), recall, charges perdurantism with being ontologically profligate in virtue of the existence of a plethora of temporal parts where we see a single persisting object. A terdurantism that is not eliminativist about ordinary objects - even a terdurantism that is restrictive about composition - will still be committed to the existence of a plethora of largely spatially and temporally scattered fusions. So terdurantism is quite ontologically profligate. On the other hand, it does allow us to reject the idea that persisting entities are composed of a series of distinct temporal parts that pass into and out of existence 'before our eyes'. At least, there is an extent to which it accomplishes this. Terduring fusions are very clearly not temporally segmented. There is no sense in which there is a series of wholly distinct entities that are related in some way to that fusion - whether as parts or not. It is also true that ordinary objects - terduring non-fusions - are not composed, at each time at which they exist, of distinct objects. Such objects have no maximal temporal parts. On the other hand, insofar as ordinary objects are (strongly) gaining and losing parts across time, they are compiled at different times, by different fusions. It is these fusions that are ontologically basic. So at each time at which a terduring non-fusion is present, it is in virtue of some distinct fusion being present at that time. While 'watching' a non-fusion across time is not watching something that is literally temporally segmented, insofar as the existence of that non-fusion is entailed by the existence of a plethora of different fusions at times, it is watching that series of distinct fusions at each of those times. It is, therefore, not obvious that the terdurantist fares all that much better in this respect than the perdurantist, but with the added cost of a bi-partite ontology of fusions and non-fusions.

\section{Instantiating Properties}

I suggested that the terdurantist might have a preferable account of property instantiation to either the perdurantist or the endurantist, hence avoiding objections (2) and (2a). The idea is that the terdurantist will not analyse the instantiation of temporary properties in terms of the properties simpliciter, of (maximal temporal) parts of persisting objects. Rather, the properties we attribute to terduring objects are indeed properties of those objects, not of their parts. Nor does the terdurantist need to reconcile her account of property talk with the notion that persisting objects are literally strictly identical across time, and hence she need not appeal to the relativisation, in some way, of properties. In this section, 
however I will argue that the terdurantist is no better off than the endurantist or the perdurantist when it comes to explicating talk of temporary properties.

How will the terdurantist understand property instantiation? Local intrinsics, naturally enough, can be understood in terms of an object having a spatial part that instantiates some property simpliciter. Temporary properties, however, must require some sort of instantiation relation. Since temporary properties are properties of the whole fourdimensional object, they cannot straightforwardly be properties simpliciter of that object, on pain of contradiction. The terdurantist could simply import the analysis already developed by endurantists, and talk of instantiating properties at times, or in temporal ways: at-t or t-ly. Then terdurantism would solve (2a), but not (2), and we would have no reason to prefer the view to endurantism. But the instantiation relation need not be temporal. Since the terdurantist is not preoccupied with the idea that persisting objects are both strictly identical to themselves at every time at which they exist and wholly present whenever they exist, she is not committed to the idea that there is something special about a three-dimensional 'slice ${ }^{25}$ of a persisting object. For it is not the case that such a slice just is a wholly present (3D) object at a particular time. Hence the terdurantist could just as well talk of having properties at space-time regions, where these regions need not correspond to some three-dimensional 'slice' of the four-dimensional worm. The region could be four-dimensionally extended. So terduring objects would instantiate temporary properties at a region, or in a particular regional manner: at- $R$, or $R$-ly. Then for any terduring object that has different properties at different times, the terdurantist must appeal to some irreducible instantiation relation: at- $R$ or $R-l y$.

It is clear, at least with respect to terduring simples, why this relation must be irreducible. In the case of local intrinsics some instantiation relation - say, instantiating a property at a location, (at- $L$ ) or, in a locational manner ( $L$-ly) - would be reducible just so long as the relation could be analysed in terms of having a spatial part at that location with that property. But this cannot be so where we are considering some terduring simple: there are no candidates for reduction for there are no parts. Likewise this is the case for terduring fusions. Precisely because such fusions are not temporally segmented, there is no prospect for reducing the instantiation relation. ${ }^{26}$ In the case of terdurtemporal part of a persisting object.

26 One helpful referee has pointed out that what at the beginning of the paper I called 
ing non-fusions, the temporary properties of such objects are in some sense reducible to the properties of the fusions that compile them at times. But since the properties of the fusions are themselves irreducibly 'regionalised', ultimately the reduction fails here too. Whenever some persisting entity has different properties at different times, it must be because it instantiates some irreducibly regionalised property, or some irreducibly regionalised instantiation relation.

Given that the terdurantist must commit herself to there being an irreducible instantiation relation, and thus to its being the case that temporary properties are never straightforwardly instantiated simpliciter, the view does not seem any improvement on endurantism.

\section{Substantivalism and Relationalism; Monism and Dualism}

Here is another reason to prefer perdurantism to terdurantism. There is a general question of what the terdurantist should say about the relations between objects, and the relations between space-time regions on the one hand, and the relations between objects and space-time regions on the other hand. Let us introduce some distinctions. Let us say that substantivilists are realists about space-time regions and, if they exist, space-time points. Relationists hold that space-time regions and points are not ontologically real: talk of space-time regions is not talk about some entity, a space-time region. Rather, talk of space-time regions is really just talk about locations in space-time, which is ultimately reducible to talk about the relative spatial and temporal distances between objects.

'weak' terdurantism is consistent with being able to reduce the instantiation of properties of a non-fusion at a time, to the properties simpliciter of the fusion that compiles it at that time. Weak terdurantism does not guarantee that one can do this, since all weak terdurantism allows is that there might exist shorter-lived objects that wholly coincide with terduring fusions at times, but are not parts of those fusions. It does not guarantee that this is the case, nor certainly guarantee the stronger claim for every temporal interval and instant at which a fusion F exists, there is some fusion $\mathrm{F}^{*}$ that exists during, or at, just that time and coincides with $\mathrm{F}$ at or during those times. A weak terdurantism with 'insufficient' coinciding fusions would still have problems because there might not be a coinciding object that exists at the relevant time or during the relevant interval, that has the relevant property simpliciter. So while weak terdurantism might have some advantages here, I have focused exclusively on strong terdurantism throughout because weak terdurantism is generally unattractive, at least as an alternative to perdurantism, insofar as it allows that there exists entities that are very much like temporal parts, albeit that they are not parts of persisting objects but are merely entities that coincide with those objects at times. 
There is another distinction that cuts across the substantivilist/relationalist distinction: the monist/dualist distinction. ${ }^{27}$ Monists hold that the relation between space-time regions and the objects that exist at those regions $^{28}$ is one of identity. Dualists hold that there exist ontologically real space-time regions such that some of those regions are occupied by objects that are distinct from those regions. Dualists are necessarily substantivilists. Monism, on the other hand, comes in two flavours: substantivilist and relational. Substantival monists think that space-time regions are ontologically real, and that objects are to be identified with those regions or the pattern of properties possessed by them. Relational monists think that only objects exist, and to the extent that we can talk about space-time regions, we are really just talking about those objects and the relations between them.

Perdurantism is consistent with all three views: substantival monism, substantival dualism and relational monism. Indeed, perdurantists have a nice story to tell regardless of the view they adopt. For perdurantism preserves a mirroring relation between objects and space-time regions. Let us say that an account of objects and their persistence mirrors an account of space-time and its regions, iff the relation that holds between regions and sub-regions of space-time is the same relation that holds between the objects that exist at those regions and sub-regions of space-time. Further, let us say that we have an exact mirroring iff for any object $O_{1}$ that occupies region $R_{1}$, it is the case that for any sub-region $R^{*}$ of $R_{1}$ that is related to $R_{1}$ by relation $R$, there is some object $O^{*}$ that exists at and only at $R^{*}$ and is related to $O_{1}$ by the $R$ relation. So if relation $R$ is the mereology relation, then we will have what Simons calls the GCP: the geometric correspondence principle, according to which any extended object has parts corresponding to the parts of the region at which the object exists. ${ }^{29}$

Consider synchronic mirroring: mirroring at a time. Perdurantists, endurantists and terdurantists can all agree that there is exact synchronic mirroring. They agree that the relation that holds between a (spatial) region $R_{1}$ at $t_{1}$, and the sub-regions of $R_{1}$ at $t_{1}$, is the part/whole

27 This terminology is from D. Lewis, On The Plurality of Worlds (Oxford: Blackwell 1986), n. 55, on 76.

28 I use the locution of objects 'existing at regions' as neutral terminology between objects being identical to the regions at which they exist, and occupying the regions at which they exist.

29 P. Simons, 'Extended Simples: A Third Way Between Atoms and Gunk,' The Monist 87 (2004) 371-85. Simons' GCP is phrased slightly differently, in terms of objecting having parts corresponding to the parts of the region the object occupies. I prefer this version, since it is neutral between monism and dualism. 
relation. So too they agree that the relation that holds between object $O_{1}$ that exists at $R_{1}$ at $t_{1}$, and the objects that exist at each of the sub-regions of $R_{1}$ at $t_{1}$, is the part/whole relation: the objects that exist at the subregions of $R_{1}$ at $t_{1}$ are spatial parts of $O_{1}$ at $t_{1}{ }^{30}$ The perdurantist also holds that there is what we might call diachronic mirroring. She holds that the relation that holds between a four-dimensional volume $R_{2}$, and the sub-regions of $R_{2}$ is the part/whole relation, while the relation that holds between the four-dimensional object $\mathrm{O}_{2}$ that exists at $R_{2}$ and the objects that exist at the sub-regions of $R_{2}$, is also the part/whole relation: in the latter case the objects that exist at the sub-regions of $R_{2}$ are temporal parts of $\mathrm{O}_{2}$. Moreover, the perdurantist can hold that this mirroring is exact: it is the same relation in both cases. Since perdurantists can straightforwardly accept the GCP, perdurantism is consistent with either monism or dualism: one can hold either that the relation between regions of space-time and four-dimensional objects is one of identity, or one of occupancy.

Terdurantists must deny that there is an exact mirroring, since they deny that there is diachronic mirroring. This means denying the GCP which in turns means denying that objects are identical to the regions at which they exist. If terdurantism is true, then monism must be false. ${ }^{31}$

Of course, one might have independent reason to think that monism is false. One might suppose that the relation between objects and the regions at which they exist must be contingent, as is attested by the fact that each have different modal properties. Objects could have existed at regions other than the regions they in fact exist at, while regions lack this property. Whatever one thinks of this objection to monism ${ }^{32}$ at the

30 This is not to say that they must agree that there is exact synchronic mirroring. Those who reject the doctrine of undetached arbitrary parts with respect to objects but not regions will deny that the mirroring is exact. Those who hold that simples are not only four-dimensionally extended, but extended in space as well will also deny that the mirroring is exact.

31 There is a difference here between an atomistic terdurantism and a gunk terdurantism. The former can accept that there is an exact synchronic mirroring, while the latter cannot. But all terdurantists deny that there is exact diachronic mirroring. Gunk terdurantists deny that there is diachronic mirroring, because they hold that some of the occupied sub-regions of any four-dimensional stream of gunk are not parts of that gunk, though they are, presumably, parts of the region that the gunk occupies. Atomistic terdurantists deny that there is diachronic mirroring, because they hold that for any extended simple $S$, there are sub-regions occupied by that simple that are not parts of the simple, though those sub-regions are parts of the region occupied by the simple. 
very least it is noteworthy that we have another metaphysical question that terdurantists are committed to answering one way, rather than any other: it seems that many doors are closed to the terdurantist.

Moreover, we might suppose that having to reject the exact mirroring principle, and specifically the GCP, is itself a cost. Again, this will depend how independently plausible one finds these principles. There are those who don't find them plausible at all.$^{33}$ It is not possible, within the space constraints, to consider the plausibility of these principles. Suffice to say that something like the GCP is, I think, fairly widely accepted, and so it would be seen by many to be at least some sort of cost to terdurantism that it must reject these principles.

So far then, we have seen both that there are a number of costs to terdurantism, and that despite appearances it does not really deliver on its promise to provide a plausible middle ground theory of persistence. But what lessons can we take from that failure?

\section{Endurantism Considered}

Endurantists deny that persisting objects are composed of temporal parts. Given this, they must inevitably develop a view that is strictly analogous to terdurantism, but with the added codicil that enduring objects are strictly identical across time, rather than being four-dimensionally extended. They too must reject the idea of instantaneous simples; they too must hold that persisting objects are fusions of, in their case, enduring simples; they too must admit that ordinary objects are not identical to such persisting fusions; they too must reject exact mirroring and the GCP; they too must accept some irreducible instantiation relation. This must be the case, because it is the terdurantist's attempt to avoid being committed to the existence of temporally segmented objects that

out by the designation 'object' as by 'space-time region', nevertheless each of these designations have attached to them different sets of counterpart relation - regionrelations and object-relations - such that not all of the region-counterparts are also object-counterparts and vice versa, thus explaining why objects and regions have different modal properties despite being identical. T. Sider, Four-Dimensionalism, 110-13.

33 P. Simons, 'Extended Simples,' 371-85; P. Van Inwagen, 'The Doctrine of Arbitrary Undetached Parts,' Pacific Philosophical Quarterly 62 (1981) 123-37; N. Markosian, 'Simples, Stuff and Simple People,' The Monist 87 (2004) 405-21. In the case of Simons and Markosian, this is at least in part motivated by a particular view about extended simples. 
pushes her to each of these claims, and this is precisely the same commitment that the endurantist makes.

Perhaps what is controversial here is that the endurantist must reject the idea that ordinary object are not identical to persisting fusions. For here, perhaps, one might be inclined to argue that while terdurantists ought not accept a temporally modified parthood relation, endurantists surely should. And if parthood is temporally modified, then we can construe ordinary objects that strongly lose parts as fusions of different particulars at different times. Hence there will be no need to appeal to an apparatus of fusions and non-fusions that are related in some mysterious way at times. The endurantist ontology will not look like the rather odd terdurantist's ontology that I described earlier.

But I think this is a mistake. The reason the endurantist should reject temporally modified parthood is not the same as the terdurantist's reason for rejecting it. What is the same is that in either case, embracing temporally modified parthood entails embracing, at a minimum, the claim that for every composite persisting object and every time at which that composite exists, there exists an instantaneous object that overlaps all and only the parts of that object at each of those times. Embracing temporally modified parthood is embracing something that is tantamount to a perdurantist ontology, minus the claim about the manner in which these instantaneous objects are related to persisting objects - minus the mereological claim. And that is surely something that endurantists want to deny. Unlike in the case of terdurantism, the endurantist does not reject such a parthood relation on the grounds that we can only fuse whole particulars, and that simples are four-dimensionally extended and hence not whole at a time. Rather, it appeals to the fact that we can only fuse distinct particulars, and that enduring particulars are strictly identical across time. For the terdurantist, it makes no sense to fuse simples at times, because there is no whole entity, at each of those times, to fuse: to be a part of the fusion in question. The reverse is true for the endurantist. Here it makes no sense to talk of fusing particulars at times, because it only makes sense to fuse distinct particulars. But there is no guarantee that the $x s$ at $t$ and the $y s$ at $t^{*}$ are not one and the same enduring things, which makes a nonsense out of mereological universalism.

That being said, it seems that the endurantist is faced with the very same problems as the terdurantist. With the exception of an appeal to irreducibly relativised properties, or an irreducible instantiation relation, these objections are not the standard objections to be found in the literature. They have nothing to do with whether objects are, or could be, strictly identical across time, or whether it makes sense to talk of objects being wholly present whenever they exist. These problems are not only novel, but more importantly, they allow us to abstract away 
from issues which, at least in part, frequently seem to come down to brute intuition on both sides.

\section{Lessons Learned}

So what are the lessons learned here? Well, the first lesson is that fourdimensionalists should be perdurantists, and that is perhaps no great surprise. But it is noteworthy that perdurance is resilient in the way it is: it is not merely that perdurantist versions of four-dimensionalism are on the whole preferable to non-perdurantist theories. Rather, it is that once one strays from perdurantism, one's theory begins to look very implausible indeed: trying to construct any version of fourdimensionalism that does not appeal to the apparatus of temporal parts is difficult, and even the most plausible of such theories is faced with problems. Moreover, these very same problems plague non-fourdimensionalist theories that are non-perdurantist. Endurantism has the same problems. This suggests that temporal parts are resilient: they are a fundamental aspect of any plausible theory of persistence.

This further suggests that many of the traditional objections to endurantism have been misplaced. For none of the problems faced by terdurantism or endurantism rest on explicating any sense in which objects are wholly present whenever they exist. Nor do they rest on attempting to make sense, in the context of Leibniz Law, of how an object with one set of parts and properties that occupies one three-dimensional region of space-time, can be strictly identical to an object with a different set of parts and properties that occupies a different three-dimensional region of space-time. Rather, the problems that endurantism faces are problems that any non-perdurantist theory faces. The true lesson of terdurantism then, is that temporal parts are resilient: any plausible theory of persistence will be a perdurantist one. We should, it would seem, all believe in temporal parts.

Received: April 2007

Revised: April 2008 